Revista Complutense de Historia de América

ISSN: $1132-8312$

http://dx.doi.org/10.5209/RCHA.56736

\title{
Duck and Cover! El impacto de la Guerra Fría en los niños estadounidenses. Una aproximación a la historiografía norteamericana sobre la infancia ${ }^{1}$
}

\author{
David Corrales Morales ${ }^{2}$
}

Recibido: 11 de enero de 2016 / Aceptado: 17 de mayo de 2016

Resumen. Desde los años sesenta, la historia de la infancia -y de los niños- ha sufrido una progresiva evolución, que ha permitido establecer una interrelación entre su objeto de estudio y ámbitos como la sociedad, la cultura o las relaciones internacionales. Este artículo aborda este amplio recorrido a través de aquellas aportaciones más relevantes centradas en las experiencias de los niños estadounidenses. Asimismo, a modo de caso práctico, el autor engloba algunas de estas líneas de estudio que han ido apareciendo en la historiografía norteamericana -familia, educación, productos de entretenimiento, etc.bajo un mismo eje temático, las repercusiones de la Guerra Fría en los más pequeños. De este modo, se aspira a proporcionar una visión -general y particular- sobre las posibilidades que ofrece esta rica disciplina historiográfica para un mayor conocimiento sobre ciertos acontecimientos y procesos históricos en los Estados Unidos.

Palabras clave: Cómic; educación; relaciones internacionales; juventud; niño; infancia; historiografía; Guerra Fría; Estados Unidos; siglo XX.

\section{[en] Duck and Cover! The Impact of the Cold War on American Kids. An Approach to the US Historiography of Childhood}

\footnotetext{
Abstract. Since the 1960s, the history of childhood -and children- has undergone a constant evolution, which has allowed for the establishment of an interrelation between its object of study and broad historical issues, such as society, culture and international relations. This article will explore the development of this academic field through the most relevant contributions focused on American kids' experiences. Similarly, as a case study, the author will bring some of these lines of research together -family, education, entertainment products and so on- in order to address a common subject matter: the effects of the Cold War on children. In this way, the article will provide an overview as well as a specific analysis of the possibilities that this historiographical discipline offers for the promotion of a better understanding of certain historical events and processes in the United States.

Keywords: Comic; Education; International Relations; Youth; Child; Childhood; Historiography; Cold War; United States; 20th Century.

Sumario. 1. Introducción. 2. De los estudios pioneros al "giro cultural". 3. "Pequeños actores" en el escenario internacional. 4. Cold War Kids, un caso concreto de análisis. 5. Conclusiones. 6. Referencias bibliográficas.

$1 \quad$ Este artículo se ha elaborado en el marco del proyecto de investigación "La modernización del sistema educativo y científico español en su dimensión internacional, 1953-1986” (Ministerio de Economía y Competitividad, HAR2014-58685-R).

2 Consejo Superior de Investigaciones Científicas (CSIC)

E-mail: david.corrales@cchs.csic.es
} 
Cómo citar: Corrales Morales, D. (2017), Duck and Cover! El impacto de la Guerra Fría en los niños estadounidenses. Una aproximación a la historiografía norteamericana sobre la infancia, en Revista Complutense de Historia de América 43, 333-354.

\section{Introducción}

A lo largo de los años sesenta y setenta, la emergencia de trabajos centrados en la infancia fue menospreciada en los círculos académicos, donde se valoraba esta novedosa disciplina como una mera tendencia pasajera, fruto de una mayor atención dada a la figura del niño tras el final de la Segunda Guerra Mundial ${ }^{3}$. Sin embargo, el recorrido de esta corriente historiográfica se ha mantenido hasta la actualidad, obteniendo un creciente protagonismo. Más allá del impacto que han generado ciertas preocupaciones sociales relacionadas con los menores -analfabetismo, delincuencia, pobreza, etc.-, una de las razones de esta evolución responde al interés despertado por aquellos individuos históricos que habían estado al margen de cualquier tipo de análisis durante décadas. Gracias a ello, distintos investigadores han subrayado la centralidad adquirida por este colectivo en un gran número de aspectos de la vida política, social y cultural, iluminando tanto las iniciativas desarrolladas por el mundo adulto como las acciones autónomas de estos sujetos en un marco no creado por ellos. Una labor que no sólo ha englobado diversas líneas de estudio -familia, educación, asistencia social, salud, productos de entretenimiento, etc.-, sino que también ha establecido lazos de unión con múltiples campos científicos, como la antropología, la sociología o la economía, que requieren una metodología diferente. Todo ello al servicio de un único objetivo: poner en cuestión la visión utópica y romántica de la infancia que ha perdurado desde siglos atrás.

En medio de este panorama tan favorable, académicos como el sociólogo Claude Javeau afirman que el refinamiento conceptual asume en esta disciplina un papel primordial en la construcción del objeto de estudio a fin de que los investigadores eviten aquellos conflictos semánticos asociados a la edad pueril y puedan delinear de una forma más clara y específica sus ámbitos de operatividad ${ }^{4}$. El término "infancia" hace referencia al período de vida comprendido entre los primeros años de una persona y la adolescencia, etapa que sirve de transición hacia la madurez. Esta fase, experimentada de manera diferente por cada niño, se encuentra modelada por un conjunto de actitudes culturales, circunstancias económicas, estructuras sociales y políticas públicas, que obedecen a un momento y lugar determinados ${ }^{5}$. Por esta razón, existen dificultades a la hora de precisar aquellos momentos clave que trazan el inicio y final de esta etapa.

El empleo de este concepto obedece a una visión de la esfera adulta en la que se perpetúan la inocencia y dependencia asociadas a este período, al tiempo que se ocultan las acciones de los actores que lo constituyen con la intención de subrayar la necesaria protección del Estado y la sociedad civil. Una perspectiva que ha dominado en esta disciplina académica durante décadas, trazando una rica línea de investigación que sólo ha sido contestada en los últimos años gracias a trabajos como

Ver Ariès, 1987; DeMause, 1974; Shorter, 1975; Stone, 1977.

Javeau, 2000: 25.

Grew, 2009: 122. 
los de Marcia Chatelain o Eileen Luhr ${ }^{6}$. Bajo este punto de vista, el uso del término "infancia" por parte del historiador hace referencia de manera exclusiva a aquellas ideas, iniciativas y representaciones en torno a este grupo social que han sido configuradas desde una óptica adulta -pedagogía, reglamento jurídico, políticas sociales, proyectos de carácter privado, producción cultural, etc. En otras palabras, se trata de una noción que recoge las ansiedades y aspiraciones que una sociedad articula en torno a los menores de edad.

El progresivo rol que ha adquirido la edad como categoría social dentro del campo académico ha fomentado que investigadores como Howard P. Chudacoff y Paula $\mathrm{S}$. Fass reivindiquen la utilización de un concepto más conveniente al objeto de estudio de esta corriente historiográfica -"niños". Su connotación, vinculada especialmente al ámbito antropológico, subraya el papel activo que desempeñaron estos sujetos, así como sus experiencias y rasgos singulares ${ }^{7}$. Frente a la constante inclinación hacia aquellos proyectos promovidos por instituciones públicas o agentes privados, este término refleja cómo los análisis centrados en los menores de edad aportan una nueva mirada sobre el escenario sociopolítico y el desarrollo de ciertos procesos históricos ${ }^{8}$.

Sin embargo, al igual que sucedía al abordar la noción de infancia, no existe unanimidad académica al precisar cuáles son las fronteras generacionales sobre las que se enmarca esta denominación. Esta cuestión resulta de suma complejidad si atendemos a la idea de que los cambios asociados a una "maduración cronológica y secuenciada" son modificables según las circunstancias de cada momento: "The chronological age at which each generation 'comes of age' moves up and down with the complex interplay of social, political, economic and environmental forces"'. Debido a ello, una definición contextual de los límites de edad de aquellos individuos conocidos como "niños" evitaría la aparición de múltiples percepciones y valoraciones erróneas que asumieran cualquier proceso de age compression desde una óptica actual $^{10}$.

Dado que la condición y concepción del niño varían históricamente, un gran número de investigadores se han escudado en la inestabilidad de esta categoría social con el fin de eludir su identificación bajo un rango de edad. No obstante, el presente análisis ha optado por delimitar este sujeto a través de la expresión middle childhood, acuñada por el propio Chudacoff. Según este historiador, el término "niños" estaría inscrito dentro del período comprendido entre los seis y doce años, estableciendo una transición entre los primeros años de vida y la adolescencia ${ }^{11}$. Esta etapa,

Chatelain, 2014; Luhr, 2015.

Javeau, 2000: 27.

Honeck - Rosenberg, 2014: 235.

Lassonde, 2011: 65.

10 El término age compression fue manejado por numerosos empresarios norteamericanos durante los años noventa. Esta expresión pretendía describir aquellos esfuerzos de la industria estadounidense que iban dirigidos a los más pequeños con la intención de que compraran y consumieran productos diseñados previamente para su uso adulto -cosméticos, películas de contenido violento, etc. Sin embargo, autores como Stephen Lassonde han empleado este concepto desde una perspectiva más general, haciendo referencia a cualquier fenómeno o acción que desafíe aquellos principios de facto sobre lo que un individuo puede -o no- hacer en una sociedad determinada en función de su edad. A pesar de ello, conviene insistir en que estas "normas" sociales se han ido modificando a lo largo de las décadas, por lo que los fundamentos concebidos años atrás difieren de los mantenidos en la actualidad. Ver Ibídem: 53.

11 Chudacoff, 2007: XV. 
marcada por la inocencia, libertad y ausencia de sexualidad, favorece un proceso de desarrollo en los menores de edad, que afecta a sus habilidades logísticas, capacidad emocional, socialización, sentido de responsabilidad, etc ${ }^{12}$. Asimismo, es una fase que, más allá del juego y el forjamiento de amistades, los niños empiezan a comprender cómo el mundo que les rodea se encuentra estructurado a través de jerarquías y categorías de diversa índole. Todas estas características han motivado la decisión de escoger principalmente estos límites de edad como los más apropiados a la hora de definir la figura del niño en este estudio.

Este refinamiento de carácter conceptual permite apreciar la emergencia y evolución de dos líneas de investigación divergentes en torno a esta disciplina historiográfica. A pesar de esta tendencia, no conviene valorar estos dos términos como categorías opuestas, sino todo lo contrario. El estudio de la infancia y de los niños se encuentra conectado bajo un mismo objetivo: proporcionar un mayor conocimiento sobre las experiencias a las que tuvieron que hacer frente los menores de edad ${ }^{13}$. Gracias a ello, se puede profundizar en temas como el contexto sociopolítico donde este grupo social vivió, las iniciativas y políticas públicas promovidas desde la esfera adulta y el rol activo que los más pequeños desempeñaron a través de sus propias acciones.

Tomando como referencia el ámbito estadounidense, las siguientes páginas examinan la evolución de esta corriente académica desde sus inicios hasta la actualidad, aunando posteriormente algunas de sus vertientes más representativas bajo un mismo objeto de estudio -las repercusiones de la Guerra Fría en los niños norteamericanos. Frente a la persistencia de viejos paradigmas y enfoques, este artículo busca reflejar las numerosas posibilidades que ofrece esta innovadora perspectiva a la hora de analizar determinados fenómenos y procesos históricos, especialmente aquellos de naturaleza internacional.

\section{De los estudios pioneros al "giro cultural"}

A partir de los años sesenta, la historiografía pretendió otorgar un significado a la nueva coyuntura que había emergido tras el final de la última conflagración mundial. Junto a las constantes transformaciones que se habían producido en el terreno cotidiano -aumento de la esperanza de vida, incremento progresivo del número de divorcios, etc.-, acontecimientos como el denominado "Mayo del 68" pusieron de relieve la existencia de fuertes relaciones de poder en niveles y categorías sociales que habían estado subestimadas hasta ese momento. Esta tendencia contribuyó a la aparición de un gran número de temas vinculados a innovadoras propuestas teóricas, entre las que se encontraron aquellas relacionadas con la familia y la edad pueril.

Una de las aportaciones más relevantes en el campo académico fue la obra de Philippe Ariès (L'enfant et la vie familiale sous l'ancien régime, 1960). Bajo su punto de vista, el proceso de escolarización, desarrollado a finales del siglo XVII, había servido como base para el establecimiento de una serie de ideas modernas vinculadas a la noción de infancia -interiorización de los principios racionales, incorporación de normas de conducta basadas en el control de los instintos, etc. Un hecho que sólo

Lassonde, 2011: 59.

13 Hawes - Hiner, 2008: 45. 
fue posible en la práctica gracias a la "complicidad sentimental" de las propias familias, caracterizadas por un repliegue en la pareja y un mayor reconocimiento de la figura del niño ${ }^{14}$. La civilización medieval había considerado la relación directa con la esfera adulta como el medio de aprendizaje más adecuado para la formación de los más jóvenes. Un pensamiento utilitario que enlazaba con el prototipo de "familia extensa" que dominaba en ese momento, determinada por la relevancia de cada uno de sus miembros como fuerza de trabajo destinada a proporcionar el sustento económico necesario. Sin embargo, coincidiendo con el final del Antiguo Régimen, la implantación de un nuevo modelo de enseñanza, cuyo espacio representativo era la escuela, restringió de manera notable el nivel de libertad y aumentó la severidad de los castigos recibidos ${ }^{15}$. A través de esta tesis principal, Ariès pretendía demostrar el peso que había adquirido esta concepción de la infancia en el imaginario colectivo de la sociedad occidental, afectando a sus hábitos y costumbres.

A pesar de que la tesis del historiador francés no tuvo gran repercusión durante los años posteriores a su publicación, su prestigio en el terreno de las ciencias sociales se acentuó durante la década de los setenta. Una gran variedad de trabajos continuaron la labor desempeñada por este estudioso, aunque intentaron priorizar como objeto de estudio las experiencias de los menores de edad ${ }^{16}$. En los Estados Unidos, frente al panorama boyante que presentaba el escenario europeo, este tipo de análisis carecieron inicialmente de unas condiciones idóneas que estimularan un interés de carácter histórico por la naturaleza de la infancia y las vivencias de los niños.

No obstante, la preeminencia de publicaciones de índole psicológica no impidió que distintos historiadores sociales -Virginia Yans-McLaughlin, John Demos, Joseph Hawes, etc.- empezasen a establecer las bases para la aparición de esta nueva disciplina en el campo académico estadounidense ${ }^{17}$. Unas aportaciones que no lograron superar la perspectiva adulta mantenida hasta ese momento, es decir, los niños seguían siendo definidos como seres dependientes, cuya identidad y comportamiento eran regulados desde arriba. Esta visión también quedaba reflejada a la hora de valorar otras etapas de la juventud. Así, por ejemplo, la obra de Joseph F. Kett (Rites of Passage: Adolescence in America 1790 to the Present, 1977), destinada a observar la evolución histórica de la adolescencia como concepto y ciclo de vida, subrayaba la emergencia de un entorno opresivo, que había sido regulado por numerosos actores -pedagogos, eclesiásticos, organizaciones juveniles, etc.- con la intención de privar la libertad de los jóvenes y favorecer una uniformización de sus experiencias ${ }^{18}$.

Los años ochenta y noventa dieron paso a una etapa de incertidumbre, en la que se produjo un debilitamiento de los viejos paradigmas historiográficos. Si bien el aumento de los problemas sociopolíticos, así como la redefinición del sistema internacional, no minaron la necesidad de un mayor conocimiento del pasado, innovadoras propuestas pusieron en cuestión los viejos esquemas interpretativos de las décadas precedentes. Los historiadores sociales habían otorgado voz a la figura del niño dentro de sus marcos institucionales -familia, escuela...-, pero sus aportaciones resultaban insuficientes para la consolidación de una disciplina que aspiraba a




proporcionarles un papel como sujetos históricos e independientes por sí mismos ${ }^{19}$. Por esta razón, la historia cultural, cuyo objetivo se basaba en revelar cómo distintos grupos sociales se definían y el significado que otorgaban a su identidad, ayudó a atenuar algunas de las limitaciones que habían estado presentes previamente. Aquellos trabajos adscritos a esta tendencia pretendieron profundizar en los detalles -como espacios u objetos materiales- como vía para comprender la infancia como un patrón cultural de la sociedad, que delineaba a los más pequeños a tenor de su edad, sexo o nivel de dependencia ${ }^{20}$. Un enfoque que, ante la escasez de fuentes primarias, no siempre consiguió documentar los testimonios y vivencias de los niños de manera directa.

Fruto de esta coyuntura, David Nasaw (Children of the City, 1985) prestó atención a las calles de las ciudades norteamericanas como lugares de trabajo y recreo para los menores de edad durante las primeras décadas del siglo XX. El análisis de novedosas fuentes -fotografías, biografías de personalidades célebres, reportajes escritos por reformadores progresistas de ese momento, etc.- permitió trazar las experiencias de aquellos jóvenes que labraron un espacio y autonomía propios en medio de un contexto urbano. A diferencia de las circunstancias erigidas a lo largo del período decimonónico, Nasaw sostenía que las relaciones sociales de mercado no habían sido particularmente duras para los niños durante esos años ${ }^{21}$. De hecho, las contribuciones económicas que este grupo social aportaba al núcleo familiar disminuyeron como consecuencia de una mejora de las condiciones sociales, situación que les permitió disfrutar del consumo de una amplia gama de productos de entretenimiento ${ }^{22}$. Este tipo de argumentos iban dirigidos a enfatizar la visión positiva que los niños tenían de su trabajo: "A pleasant interlude between a day's confinement in school and an evening in cramped quarters at home"23.

Frente a aquellos trabajos que buscaban trazar la identidad de este colectivo social en función de aquellos espacios o materiales que habían entablado un contacto directo con ellos, otras aportaciones académicas abordaron algunos fenómenos que englobaban muchos de estos aspectos culturales bajo una única categoría. Uno de los casos más ilustrativos fue el juego, definido como el conjunto de actividades de recreo que dotan de una retribución a nivel intelectual, emocional, físico y social. Este objeto de estudio, cuya popularidad se vio acrecentada ante los continuos debates sobre su aplicación en el campo pedagógico, se convirtió en la pieza central de la obra de Howard P. Chudacoff (Children at Play, 2007), que ahondaba especialmente en los cuatro elementos sobre los que se desarrollaban sus cualidades lúdicas y utilitarias: medio, cultura material, individuo y libertad. A lo largo de su investigación, este historiador remarcaba el peso que determinadas características -edad, sexo, clase social, etc.- ejercían sobre los gustos, estilos o propiedades del juego ${ }^{24}$. Más allá de su diversidad, sus dinámicas sirvieron como un medio de afianzamiento de la autonomía personal. No obstante, en múltiples ocasiones estas aspiraciones menguaron ante la injerencia de un mundo adulto, que consideraba vital un ejercicio de control y

\footnotetext{
Fass, 2007: 5.

Ibídem.

Nasaw, 1985: 61.

Ibídem: 196.

Ibídem: 47.

Chudacoff, 2007: 2.
} 
supervisión $^{25}$. Una situación que contribuía a apoyar la tesis de que la visión de una infancia feliz y libre no era más que una utopía.

Junto a aquellos análisis asociados a la historiografía de la infancia -y de los niños-, el auge del denominado "giro cultural" también se ha ido plasmando desde los años noventa en el aumento de una serie de líneas de estudio que, pese a estar dotadas de un marco teórico-metodológico propio, confluyen con algunos de los objetivos fijados por esta disciplina. Sin embargo, la autoría de estos trabajos, centrados fundamentalmente en diferentes productos culturales dirigidos a la esfera infantil, responde a una amplia heterogeneidad académica -antropólogos, sociólogos, lingüistas, etc. Un aspecto que, a día de hoy, contribuye a complementar el punto de vista aportado por el historiador, aunque exige cierta cautela a la hora de examinar sus aportaciones.

Si bien el balance sintético que ofrece este artículo impide abordar estos fructíferos campos de estudio en detalle, conviene detenerse brevemente en dos de ellos. En primer lugar, como consecuencia de un resurgimiento del interés por el cómic a través de distintas iniciativas -reediciones de revistas infantiles, convenciones internacionales, adaptaciones cinematográficas y televisivas, etc.-, en los últimos años se ha producido un fuerte movimiento de reivindicación, catalogación y análisis en torno a este tipo de narrativa popular. A pesar de que muchas son las polémicas surgidas en la búsqueda de una descripción clara y concisa que reúna las características más comunes de este producto cultural -estilo y forma, objetivos, valor comercial, etc.-, ello no ha impedido la proliferación de numerosos trabajos que, procedentes de un amplio marco teórico -sociología, semiótica, teoría de los medios de comunicación, etc.-, han profundizado en su contenido ${ }^{26}$.

Dentro del rico abanico de perspectivas empleadas, se ha acentuado el interés por estudiar los cómics como "productos de su tiempo". Esta aproximación analítica ha ayudado a precisar cuáles eran las actitudes que prevalecían hacia ciertos asuntos en un período determinado. En el libro War, Politics and Superheroes (2011), el investigador Marc DiPaolo examinó cómo los retratos de diversos superhéroes -Batman, Superman, X-Men, etc.- se habían modificado a lo largo del tiempo, plasmando los temas e ideas preponderantes de cada etapa. De esta manera, el autor pretendía reconstruir los mensajes políticos que habían predominado en las aventuras de estos personajes así como su influencia en la opinión pública estadounidense. No obstante, la persecución de estos objetivos conllevaba enormes dificultades, pues el contenido de los cómics se había ido amoldando a las circunstancias de cada momento y su subtexto había obedecido a una extensa gama de factores condicionantes -autor, lector, editorial, coyuntura económica, etc ${ }^{27}$.

En segundo lugar, el análisis del juguete industrial ha proporcionado información sobre la interacción establecida entre niños, padres y comercio ${ }^{28}$. Este producto puede ser definido como "un bien de consumo inmediato fabricado por adultos y adquirido esencialmente también por adultos para ser utilizado por los más pequeños" 29 . A lo largo de las décadas, la principal función de los juguetes ha sido la construcción de una representación a escala reducida del mundo adulto, que contribuyese a la progre-




siva incorporación y adaptación de los niños dentro de esa realidad. Por esta razón, su estudio no sólo da a conocer los cambios significativos que ha experimentado la noción de infancia, sino también el impacto social de la actual sociedad de consumo.

Enmarcado en esta línea temática, la obra de Gary Cross (Kid's Stuff, 1997) incidió en las tensiones generacionales que habían emergido en torno al uso de determinados juguetes norteamericanos, mostrando cómo un gran número de cambios socioculturales -aparición de grandes corporaciones, utilización de la televisión como instrumento publicitario, etc.- habían favorecido que estos productos culturales perdiesen su papel como vínculo paterno-filial en beneficio de una mayor libertad e imaginación de los niños ${ }^{30}$. Un argumento que convenía matizar resaltando algunas de las persistencias que se habían mantenido entre los juguetes de ayer y de hoy, ya que tanto la economía de consumo como la naturaleza cambiante de la cultura popular eran características que habían estado presentes en los Estados Unidos desde las primeras décadas del siglo $\mathrm{XX}^{31}$.

La muñeca, portadora de mensajes de género, se ha convertido en el artículo que más atención ha recibido por parte de los académicos. Desde un enfoque antropológico, Margaret Jacobs profundizó en el significado y valor que las niñas indias habían dado a este bien cultural, utilizado por educadoras y misioneras estadounidenses como un eficaz instrumento para la adquisición de habilidades domésticas. Este trabajo demostró claramente la resistencia que los menores de edad podían ejercer con el fin de conservar su identidad y bagaje cultural, aspecto ejemplificado en algunas de las fotografías utilizadas como fuentes primarias ${ }^{32}$. Asimismo, Miriam Forman-Brunell se centró en las ambigüedades de la célebre muñeca Barbie, cuyos modelos reflejaban una constante ambivalencia entre los valores tradicionales y los nuevos procesos de cambio social. Una tendencia que fue consecuencia de las circunstancias sociales de los años sesenta y setenta, así como de las propias experiencias de su creadora ${ }^{33}$. Según Forman-Brunell, Barbie se convirtió en la representación material de Ruth Handler, cuya vida había oscilado entre la emancipación y la sumisión en un momento histórico donde la mujer todavía se encontraba recluida en el ámbito doméstico ${ }^{34}$.

\section{3. "Pequeños actores" en el escenario internacional}

Desde comienzos del siglo XXI, la convergencia entre la figura del niño y las relaciones internacionales ha generado una fructífera línea de investigación. Esta intersección académica entre dos campos de estudio ha sido resultado de la confluencia en el tiempo de dos tendencias historiográficas. Por un lado, el análisis de la infancia desde un enfoque social o cultural no ha evitado que en los últimos años se haya acentuado el interés por aquellas instituciones -públicas y privadas- orientadas a la promoción del bienestar de los más jóvenes.

Dentro del ámbito académico estadounidense, la historiadora Marilyn Irvin Holt (The Orphan Trains: Placing Out in America, 1992) examinó el proceso de emi-

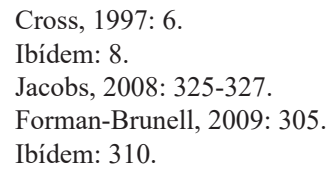


gración y reasentamiento de más de 200.000 niños norteamericanos en hogares de acogida dispuestos por todo el país entre 1850 y 1930. Si bien las constantes críticas, así como la profesionalización de los servicios sociales, pusieron fin a esta iniciativa, su promoción por parte de organismos privados como la New York's Children Aid Society se convirtió en un claro símbolo de la emergente conciencia que se había instaurado en la sociedad civil en relación al fomento de unas condiciones idóneas para el desarrollo de este colectivo ${ }^{35}$. Otra contribución más reciente es la obra de Kriste Lindenmeyer (The Greatest Generation Grows Up, 2005), donde abordaba los años de la Gran Depresión como una etapa clave en la constitución de una noción moderna de infancia, aspecto ejemplificado en la promoción de diversas medidas por parte del Gobierno federal o en la comercialización de productos de entretenimiento dirigidos a los menores ${ }^{36}$.

Por otro lado, los tradicionales planteamientos metodológicos y epistemológicos de la historia diplomática han sido rebasados por novedosos enfoques, que aspiran a conseguir una comprensión más objetiva sobre diferentes fenómenos y procesos históricos. Fruto de esta coyuntura, la perspectiva transnacional se ha convertido en una herramienta para explorar la relevancia de aquellos grupos, personalidades e ideas que, trascendiendo las propias fronteras nacionales, sirvieron para estrechar los lazos entre diferentes países y pueblos ${ }^{37}$. A su vez, el peso adquirido por el componente cultural ha impulsado temas tan variados como el turismo, el deporte, la migración o la música, cuyos análisis han contribuido a subrayar tanto las transmisiones culturales de carácter informal como las tareas desempeñadas por múltiples actores no estatales $^{38}$. Unos planteamientos que no cuestionan el peso del poder gubernamental en cualquier tipo de interacción global, sino que lo examinan desde un contexto más amplio y complejo.

La unión de ambos campos de investigación ha dado como resultado tres vías de estudio: la repercusión de ciertos fenómenos globales -guerras, migraciones, etc.- en las experiencias de los niños desde una óptica social, la aplicación de las denominadas "políticas de la infancia", promovidas por el Estado como instrumentos estratégicos al servicio de sus propósitos en el exterior, y la participación -o resistencia- activa de los menores de edad en determinadas iniciativas transnacionales. Todas ellas se asientan sobre tres principios, que se han convertido en los ejes vertebradores de cualquier investigación realizada hasta la fecha. Primero, el niño fue un participante y actor histórico del sistema internacional. Segundo, la infancia, símbolo de cambio y renovación, se convirtió en un instrumento estratégico de la diplomacia occidental, con el que se pretendía universalizar una serie de valores y establecer unos vínculos de amistad con otras sociedades. Tercero, más allá del protagonismo otorgado al niño en la ejecución de distintas acciones, sus intereses y deseos se superpusieron a cualquier objetivo político, situación que acabó minando muchas de las campañas y actividades fomentadas por organismos públicos y privados ${ }^{39}$.

Dentro de la primera línea temática, Lisa L. Ossian (The Forgotten Generation, 2011) analizó la manera en que la Segunda Guerra Mundial amenazó e intensificó cualquier dimensión de la vida de los jóvenes norteamericanos. El espíritu militar

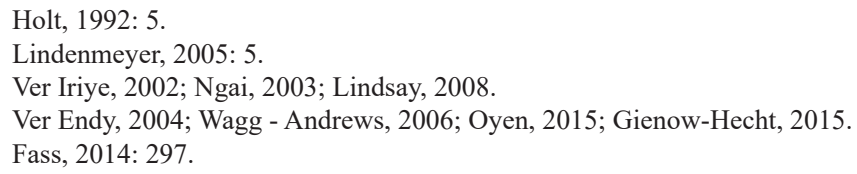


de muchos juegos, la proyección de noticieros y películas bélicas los sábados por la mañana, la recolección de materias primas o el trabajo en granjas y fábricas locales ante la escasez de mano de obra sólo son algunos de los ejemplos que plasman cómo los niños eran conscientes de las circunstancias de ese momento ${ }^{40}$. Esta "generación olvidada", empleando el apelativo acuñado por la historiadora, no recibió protección alguna de sus progenitores, sino más bien todo lo contrario. Conscientes de la necesaria movilización de los más pequeños en tiempos de guerra, sus padres les animaron a desarrollar un fuerte sentimiento patriótico así como un profundo sentido de responsabilidad en las tareas desempeñadas ${ }^{41}$.

Más allá de los conflictos bélicos, existieron fenómenos transnacionales que alteraron notablemente las experiencias de la población infantil. El tradicional interés de diversos historiadores norteamericanos por la migración de distintos grupos étnicos -Marvin Lazerson, Reed Ueda, Joel Perlmann, etc.- ha permitido resaltar sus efectos sobre la educación y movilidad social de los más pequeños. Así, por ejemplo, los alumnos inmigrantes conseguían peores resultados escolares que aquellos jóvenes cuyos padres habían nacido en los Estados Unidos ${ }^{42}$. Un planteamiento que comienza a ser matizado como consecuencia de las recientes aportaciones realizadas por algunos sociólogos y pedagogos: "Immigrant children are healthier, work harder in school, and have more positive social attitudes tan their nonimmigrant peers" 43 . Sin duda, esta afirmación invita a plantearse en el futuro cuáles fueron los factores y circunstancias que socavaron la capacidad académica de estos niños en el pasado.

En cuanto a la segunda vía de investigación, las "políticas de la infancia" (politics of childhood) pueden ser definidas como el despliegue estratégico de un conjunto de representaciones infantiles de amplio calado emocional en la búsqueda de un mayor poder y recursos, acompañado de iniciativas que involucran a los menores de edad con el fin de favorecer una serie de objetivos políticos, sociales y culturales ${ }^{44}$. Así pues, esta maquinaria oficial emplea la figura del niño como una mera herramienta para sus intereses, ya sea recurriendo a su imagen o implicándole de manera directa.

El uso del menor como representación simbólica busca generar una respuesta en el espectador, apelando a la condición inocente de la edad pueril para movilizar de manera subjetiva a la población receptora y favorecer la aceptación de ciertas ideas ${ }^{45}$. Margaret Peacock (Innocent Weapons, 2014) analizó la creación de una iconografía similar en torno al niño por parte de los Gobiernos estadounidense y soviético ante la necesidad de instaurar una aquiescencia -doméstica e internacional- que favoreciese su liderazgo a lo largo de la Guerra Fría. El futuro y progreso de la nación, la crítica al enemigo o el miedo a una posible infiltración ideológica son algunas de las metáforas empleadas en las visiones fabricadas por ambos países durante las primeras décadas ${ }^{46}$. No obstante, este consenso de carácter simbólico comenzó a ser contestado a partir de los años sesenta, cuando distintos actores privados desplegaron una nueva visión del menor, que criticaba la escasa protección recibida por parte de los Estados $^{47}$.

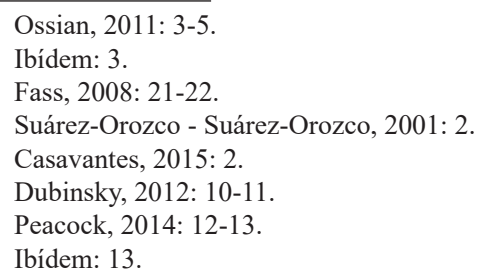


Frente al uso de imágenes propagandísticas, han sido numerosos los proyectos que han implicado a los jóvenes de manera directa. Tomando como referencia el mismo período examinado por Peacock, Joël Kotek reiteró que la infiltración sistemática de autoridades soviéticas en organismos internacionales había acabado promoviendo la financiación de diferentes organizaciones juveniles por parte de la Central Intelligence Agency $(\mathrm{CIA})^{48}$. No obstante, otros investigadores han decidido prestar atención a otras iniciativas previas. La tesis de Gabriel N. Rosenberg (Breeding the Future, 2011) tomó como objeto de estudio los llamados 4-H Clubs, administrados por el US Department of Agriculture. Para ello, esbozó su recorrido desde su creación, fruto de las reformas emprendidas a lo largo de la etapa progresista, hasta su papel como movilizador del mundo rural durante el New Deal. A través de estas asociaciones, el Gobierno federal expuso innovadoras técnicas relacionadas con la economía doméstica y la agricultura científica con el objetivo de ser probadas y perfeccionadas antes de su aplicación en territorios latinoamericanos y asiáticos durante la segunda mitad de la centuria ${ }^{49}$.

Respecto a la tercera -y última- línea temática basada en la intersección entre la figura del niño y las relaciones internacionales, no han sido pocos los historiadores que en los últimos años han fomentado constantes debates sobre cómo la juventud había conformado procesos y acontecimientos históricos bajo la premisa de ampliar la visión reduccionista centrada en la esfera política. Sus acciones no sólo generaron lazos que traspasaron las propias fronteras nacionales, sino que en ocasiones se superpusieron a los intereses de las élites dominantes ${ }^{50}$.

Un gran número de obras, tanto académicas como divulgativas, han abordado la trayectoria de determinadas organizaciones juveniles norteamericanas -Boy Scouts of America, Young Men's Christian Association, etc.-, ahondando en temas tan dispares como las labores desempeñadas por sus "padres fundadores", la evolución de sus principios éticos o las tensiones entre sus compromisos locales, nacionales y globales ${ }^{51}$. Sin embargo, conviene incidir en la resistencia ejercida por muchos menores ante la puesta en práctica de algunas actividades que requerían su participación. Una iniciativa privada que no logró cumplir sus propósitos iniciales fue la visita que algunas niñas estadounidenses realizaron a México a finales de los años cincuenta con el fin de disfrutar de una estancia en "Nuestra Cabaña", uno de los centros más importantes de la asociación Girl Scouts destinado al encuentro entre miembros de diferentes naciones. Si bien tras el final la Segunda Guerra Mundial esta organización adoptó una conciencia global que amplió las labores femeninas gracias a la incorporación de una agenda internacional, el estudio de la experiencia mexicana por Marcia Chatelain ha reflejado que este compromiso no fue asumido por las jóvenes norteamericanas, las cuales pensaban que ese viaje era un mero descanso vacacional, y no una oportunidad para establecer vínculos transnacionales con otras compañeras. A raíz de este grave malentendido, estas niñas desafiaron la autoridad de las coordinadoras, al tiempo que flirteaban con algunos muchachos de la localidad ${ }^{52}$. De este modo, sus propios intereses se antepusieron a su papel como "embajadoras" de su país.

\footnotetext{
Kotek, 2003.

Rosenberg, 2011.

Honeck - Rosenberg, 2014: 236-237.

Ver Wills, 2009; Scott - Murphy, 2010.

Chatelain, 2014: 265-266.
} 
Para finalizar este sintético recorrido historiográfico, el fenómeno de la globalización, caracterizado como una expansión e intensificación de las actividades, relaciones e interdependencias a escala mundial, ha hecho discurrir por nuevas vías la historia de la infancia -y de los niños. El recurso a una perspectiva global aspira a romper el dominio inexcusable de una visión occidental, incidiendo en cómo los más pequeños han ido adoptando una identidad específica como ciudadanos del mundo $^{53}$. De hecho, autores como Paulas S. Fass (Children of a New World, 2007) han defendido que el examen de aquellas acciones llevadas a cabo por los jóvenes en distintos lugares puede convertirse en un eficaz instrumento que aporte pruebas empíricas sobre la consolidación de una sociedad mundial ${ }^{54}$. En definitiva, un novedoso enfoque que permite inaugurar caminos inexplorados para esta disciplina historiográfica.

\section{Cold War Kids, un caso concreto de análisis}

A pesar de que las diferentes aportaciones de la historia de la infancia en el ámbito académico estadounidense han contribuido a otorgar un mayor conocimiento sobre las experiencias de los más pequeños a través de la sociedad, la cultura o las relaciones internacionales, son escasas las ocasiones en las que se ha podido valorar un mismo tema estableciendo una interrelación entre todos aquellos enfoques previamente mencionados. Por esta razón, el interés que ha generado -y sigue generando- el período de la Guerra Fría en la historiografía norteamericana ofrece una oportunidad única de mostrar las posibilidades que brinda esta corriente historiográfica a la hora de examinar acontecimientos y procesos históricos en los Estados Unidos. Este último apartado entabla un diálogo entre aquellas líneas de estudio más representativas de la disciplina -familia, educación, productos de entretenimiento, políticas gubernamentales, etc.- con la intención de reconstruir la vida de los niños norteamericanos durante los años cincuenta y sesenta, momento en que todas sus facetas estaban condicionadas por la creciente escalada de tensión entre los Estados Unidos y la Unión Soviética.

El final de la Segunda Guerra Mundial dio paso a una serie de transformaciones en campos como la legislación o la cultura de masas, que no sólo alteraron las prácticas y costumbres de los menores de edad, sino también la concepción que dominaba sobre la infancia en ese período ${ }^{55}$. A pesar de que esta coyuntura no vino acompañada de una perturbación del proceso imaginativo y, por consiguiente, del propio juego, sus efectos se hicieron muy evidentes tanto en el entorno sociocultural de los jóvenes como en las relaciones de sociabilidad con el mundo adulto ${ }^{56}$.

En medio de un panorama marcado por una notable sensibilidad hacia este colectivo, la difusión del denominado "paradigma del desarrollo", basado en la convicción de que la salud psicológica era resultado de un proceso de maduración secuenciado a lo largo de distintas fases, contribuyó a replantear los límites de edad sobre los que

\footnotetext{
Fass, 2008: 26-27.

Fass, 2007: 10.

Fass - Grossberg, 2011: X-XI.

Mintz, 2011: 41.
} 
se articulaba el crecimiento -físico y psicológico- del niño ${ }^{57}$. Este aspecto, sumado al aumento de los índices de natalidad y a una defensa acérrima de la estructura familiar, conllevó a corto plazo un reforzamiento de las categorías de género desde la juventud: "Boys would be prepared to defend the United States from threats to freedom, while girls would be readied for domestic responsibilities" ${ }^{\prime 58}$. No obstante, este intento de contención bajo esquemas tradicionales no impidió la aparición de fuertes tensiones generacionales, que rebasaron la esfera familiar a partir de los años sesenta.

Dentro de las relaciones paterno-filiales, la sociedad norteamericana era consciente de que la figura del padre cumplía un papel de suma relevancia como testigo del paso de sus hijos hacia la adolescencia, especialmente en el caso de las niñas. Finalizada la guerra, los cambios demográficos y la difícil reintegración del padre en la estructura familiar hacían peligrar este principio, motivo por el que un gran número de psicólogos y sociólogos se esforzar en explicar la contribución paterna a la crianza de sus vástagos: "Although girls identified directly with their mothers, their fathers served as an exemplar of the kind of man they would one day marry, and thus fathers should think of themselves as their, "daughters' first boyfriend" 59 .

Gracias a ello, frente a una posible erosión del poder parental, la relación entre padre e hija comenzó a ser comprendida en la cultura popular -campañas publicitarias, literatura de ficción, etc.- a través de un significado sexual, que otorgó al progenitor una identidad distintiva, capaz de reconfigurar su rol preeminente en la vida de una menor cada vez más independiente ${ }^{60}$. Sin duda, esta expresión real emergía ante la incapacidad de aceptar una mayor autonomía de los jóvenes.

El ámbito familiar también fue testigo de la importancia que adquirió el dormitorio del menor como lugar autónomo donde proyectar la personalidad individual. Más allá de una coyuntura socioeconómica favorable durante los años cincuenta, esta tendencia vino favorecida por algunos agentes. Así, por ejemplo, diversos pedagogos enfatizaron la necesidad de que los niños disfrutasen de un mayor nivel de privacidad. Según estos expertos, el derecho a esta intimidad, materializado en una habitación propia, evitaba conflictos en el hogar así como conductas inapropiadas -entre otras, dependencia materna o incesto ${ }^{61}$. Otro factor relevante fue el auge de una cultura de masas destinada al público infantil. La proliferación de múltiples aparatos electrónicos -radio, teléfono, televisión, etc.- contribuyó a la consolidación de una estancia exclusiva de entretenimiento y sociabilidad, amortiguando así el peso que habían adquirido ciertos espacios públicos ${ }^{62}$. Si bien fueron pocos los que cuestionaron las ventajas asociadas a la instauración de este cuarto, ello no evitó la permanencia de determinadas inquietudes con respecto a su carácter segregador, considerado una amenaza para la estabilidad familiar y social ${ }^{63}$.

En cuanto a aquellos temas que rebasaron la frontera doméstica, conviene señalar la enorme repercusión que tuvo el movimiento por los derechos civiles. A partir de 1954, American Bandstand, uno de los espectáculos televisivos de mayor popularidad entre el público infantil y adolescente de Philadelphia, llevó a

\footnotetext{
Lassonde, 2011: 52 .

Mintz, 2011: 43.

Devlin, 2005: 7.

Ibídem: 3 .

Reid, 2012: 432-435.

Ibídem: 428.

Ibídem: 438.
} 
cabo una política de exclusión sistemática de todos aquellos seguidores de origen afroamericano que querían asistir como espectadores. Una medida, prolongada hasta 1964, que obedecía a la prioridad de sus productores y patrocinadores, quienes perseguían la consolidación de un mercado de consumo destinado exclusivamente a jóvenes "blancos" 64 . Mostrando una indiferencia hacia los conflictos raciales que azotaban diversas ciudades norteamericanas, este tipo de entretenimiento sirvió para sustentar aquellas actitudes y políticas que limitaban el acceso de las personas "de color" a la educación, la vivienda y los servicios públicos ${ }^{65}$. Por otro parte, las ideas $\mathrm{y}$ visiones en torno a la infancia fueron empleadas por grupos de derechos civiles, valorando la juventud como un instrumento que podía ayudar a lograr un mayor apoyo a favor de este movimiento de protesta: "Childhood was a sacred state that needed to be protected and that racial discrimination obviously violated" 66 . A su vez, fueron numerosos los escolares que desempeñaron una participación activa en medio de esta reivindicación, aspecto ejemplificado en la conocida como Birmingham Children>s Crusade (1963).

A pesar de las consecuencias que provocaron estas cuestiones sociales a lo largo de la segunda mitad del siglo XX, ningún fenómeno o proceso histórico generó tanto impacto en la vida de estos niños como la Guerra Fría. El enfrentamiento entre los Estados Unidos y la Unión Soviética no respondía a las características propias de una conflagración bélica (shooting war), pues era en gran medida un conflicto de naturaleza ideológica, resultado de un profundo choque entre distintos valores y creencias ${ }^{67}$. Sus repercusiones en la esfera infantil se hicieron patentes en tres campos diferentes: las políticas domésticas, las iniciativas impulsadas por algunas organizaciones privadas y los productos culturales.

Aparte de la difusión de su modelo político y económico a otros territorios para asegurar un mayor número de aliados, la potencia estadounidense se vio en la necesidad de inculcar una serie de principios entre los más pequeños, que garantizasen el futuro de sus instituciones políticas y el predominio del estilo de vida americano frente al enemigo comunista. Una campaña en la que colaboraron un amplio abanico de actores -padres, profesores, asistentes sociales, médicos, periodistas, productores de televisión, etc.- bajo dos únicas premisas: enseñar qué significaba ser un respetado ciudadano norteamericano y explicar los fundamentos sobre los que se sustentaba la superioridad de este país ${ }^{68}$. No obstante, tal y como ha señalado la investigadora Ann Marie Kordas, resulta paradójico que los destinatarios de este adoctrinamiento fuesen a largo plazo los primeros en mostrar su firme oposición a la Guerra Fría a partir de los años sesenta, rechazando la persistente polarización entre "buenos" y "malos": "America's youth became sceptical to official pronouncements [...] it was America's success at raising boys and girls who learned their lessons too well that led the US to relinquish the battlefield" 69 .

Durante las primeras décadas del conflicto, el bienestar del niño también se instauró como un elemento fundamental en el imaginario político estadounidense, ya que este sujeto iba a convertirse en el principal responsable de preservar la sociedad

\footnotetext{
Delmont, 2012: 2 .

Ibídem: 49.

Schweinitz, 2009: 82 .

Kordas, 2013: 2.

Ibídem: 9-10.

Ibídem: 158.
} 
democrática y los ideales de la nación. Dado que muchos de los problemas dominantes -escasez de profesores, incremento de la delincuencia infantil, etc.- eran demasiado complejos y costosos, el papel del Gobierno federal se incrementó progresivamente. Esta situación no evitó cierta oposición por parte de las autoridades locales y estatales, temerosas de una pérdida de autonomía en sus respectivos territorios ${ }^{70}$.

Dentro de aquellos proyectos promovidos desde Washington, una de las actividades más populares fueron las llamadas White House Conferences on Children and Youth. Estos simposios incidieron en los problemas que afectaban a los menores de edad, proporcionando medidas para su solución. Entre los temas abordados por los grupos de trabajo constituidos, figuraban la discriminación racial, la vivienda, la incorporación de la mujer al mundo laboral o los peligros de la televisión ${ }^{71}$. Otras iniciativas de amplio calado estuvieron orientadas a la salud e higiene de los más pequeños, garantía para la seguridad y economía del país. La campaña nacional de vacunación contra la polio, la promoción del ejercicio físico o el control de productos químicos en el abastecimiento de los alimentos fueron algunas de las labores más prolíficas. Análogamente, se promovieron políticas nacionales que pretendían contrarrestar el riesgo de exclusión social mediante un aumento del número de adopciones y el fortalecimiento de la estructura familiar ${ }^{72}$.

Sin embargo, esta respuesta oficial a la amenaza comunista se materializó especialmente en el campo educativo. Por un lado, se emplearon diversos medios con el objetivo de preparar a los jóvenes ante una posible guerra nuclear: proyección del cortometraje Duck and Cover, distribución de panfletos en las escuelas, etc ${ }^{73}$. Por otro, se atendieron algunas necesidades básicas de manera selectiva. Prueba de ello es que la ayuda federal, destinada a resolver la carencia de profesores y aulas durante los años de posguerra, fue dirigida exclusivamente a los distritos escolares que habían padecido un aumento de su población ante la emergencia de nuevas instalaciones militares ${ }^{74}$.

Más allá de estas medidas, la coyuntura de la Guerra Fría, principalmente desde el lanzamiento del satélite Sputnik en 1957, impulsó disposiciones legales como la National Defense Education Act (1958), la cual concedió becas de estudio, seminarios de verano orientados a maestros y equipos científicos para los colegios ${ }^{75}$. Junto a estas decisiones de índole oficial, la situación internacional acabó motivando que el modelo educativo norteamericano se sometiese a un proceso de revaluación por parte de los círculos políticos e intelectuales, quienes cuestionaban tanto los principios del pragmatismo filosófico de John Dewey como determinados aspectos de naturaleza progresista bajo la convicción de que en ese momento no obedecían a los intereses de la nación ${ }^{76}$.

La politización de los niños estadounidenses también fue fomentada a través de diferentes organismos privados, los cuales priorizaron la participación de estos infantes de una manera activa. Uno de los casos más ilustrativos fue el de la organización

\footnotetext{
Holt, 2014: 150 .

Ibídem: 12-13.

72 Sobre aquellas iniciativas oficiales dirigidas a la salud y asistencia social del menor, ver capítulos III y IV, "The Delinquent, the Dependent, and the Orphaned" y "A Healthier Generation", en Ibidem: 82-146.

73 Kordas, 2013: 81-82.

74 Holt, 2014: 52.

75 Dentro de aquellos trabajos que abordaron este tipo de medidas como respuesta al enemigo comunista durante los años cuarenta y cincuenta, destacan: Barksdale, 1981; Foster, 2000.

76 Para un mayor conocimiento de esta polémica, ver Hartman, 2008.
} 
evangélica Youth for Christ (YFC), que animaba a defender la unión de un mundo libre y democrático frente a la Unión Soviética. Bajo un "imaginario global" que asumía aspectos de la llamada "teoría de la modernización" mentar el envío de jóvenes cristianos a países en desarrollo con la intención de que trabajasen en campos como la enseñanza o la ingeniería ${ }^{78}$. De este modo, estos activistas no sólo se convertían en baluartes contra el comunismo, sino también en heraldos de la innovación. No obstante, este proyecto transnacional produjo consecuencias no deseadas a partir de la década de los sesenta. La creación de un diálogo intercultural con otros territorios y el desencanto producido por aquellas promesas basadas en un progreso liderado por los Estados Unidos acabaron provocando que algunos de estos voluntarios criticasen la política exterior norteamericana y optasen por una mayor atención a las demandas y necesidades de esas regiones, relegando a un segundo plano aquellos objetivos vinculados al enfrentamiento entre Washington y Moscú ${ }^{79}$.

En último lugar, la tensión entre los Estados Unidos y la Unión Soviética tuvo una fuerte repercusión en la cultura de masas, la cual transmitió al público infantil una serie de imágenes y temas relacionados con este enfrentamiento ideológico. Ningún producto de entretenimiento plasmó de manera tan reiterada la lucha contra los soviéticos como los cómics, caracterizados por la defensa acérrima de la superioridad norteamericana así como por una representación estereotipada del enemigo comunista. Un panorama que se fue modificando con el transcurrir de las décadas.

Dentro de un amplio abanico de superhéroes, el Capitán América, figura de marcado carácter propagandístico a lo largo de la Segunda Guerra Mundial, se mantuvo como un símbolo de los ideales de los Estados Unidos hasta su desaparición temporal a mediados de los años cincuenta ${ }^{80}$. Desde una perspectiva diferente, la figura de Iron Man se convirtió en la personificación de la industria militar norteamericana, estableciendo un equilibrio entre los principios tradicionales, las dinámicas de la Guerra Fría y la innovación científico-tecnológica, fruto del libre comercio. Este último aspecto fue un asunto recurrente con el que se pretendía concienciar a los lectores de las ventajas que podían aportar las grandes corporaciones ${ }^{81}$. Análogamente, temas como la posible infiltración soviética dentro de las instituciones políticas o el uso de tácticas como el denominado "lavado de cerebro" permearon las aventuras de numerosos personajes populares -los Cuatro Fantásticos, Hulk, Ant Man, etc ${ }^{82}$. A pesar de esta situación, conviene señalar excepciones como las historietas bélicas de Harvey Kurtzman, quien rechazó cualquier tipo de prejuicio bajo la intención de humanizar ambos bandos del conflicto ${ }^{83}$.

77 Las formulaciones de esta teoría, desarrollada por pensadores norteamericanos como Karl Mannheim o Walt W. Rostow, se convirtieron en el andamiaje de aquellas políticas estadounidenses -de naturaleza técnica y financiera- que intentaban trasladar sus valores e instituciones a las sociedades del denominado "Tercer Mundo" con el objetivo de favorecer un amplio proceso de transformación, a través de, por ejemplo, alfabetización, urbanización, industrialización o crecimiento económico: "La modernización se identificaba con la asunción del modelo de progreso occidental -a imagen y semejanza de una visión idealizada de Estados Unidos-, adquiriendo categoría de 'hecho universal'. El Estado del desarrollo venía a ser la versión exportable a los países atrasados del Estado del bienestar, que de esta forma resultarían a su vez menos sensibles a la subversión comunista". Delgado Gómez-Escalonilla, 2015: 291.

78 Luhr, 2015: 297 y 309.

79 Ibídem: 313-314.

80 Thompson, 2013: 106-108.

81 Chambliss, 2013: 168.

82 York, 2012: 209-210.

83 Ver Field, 2012: 45-54. 
Más allá de las viñetas, la influencia que los cómics estaban ejerciendo en los más pequeños no pasó desapercibida para determinadas personalidades, que exigían una regulación de su contenido al considerar esta situación como un "desarme moral", que deterioraba los valores y creencias de los niños ${ }^{84}$. Sin embargo, es necesario matizar que la mayoría de estas críticas no reprobaban aquellos mensajes relacionados con el enfrentamiento ideológico, sino más bien el componente racista que dominaba en sus páginas, un grave peligro para un país que alardeaba de ser el máximo defensor de la democracia y de los derechos individuales a nivel mundial. Por esta razón, evitando que en el futuro pudiese establecerse un dominio federal sobre esta materia, la Comics Magazine Association of America (CMAA) emitió su propio Comics Code (1954), es decir, una lista de directivas encargadas de regular -o eliminar- todo elemento sexual, violento o racista que apareciese en estos tebeos. Una medida que no impidió la permanencia de múltiples estereotipos, especialmente en el caso de minorías étnicas ${ }^{85}$.

A partir de los años sesenta, los adversarios soviéticos dieron paso a otros problemas de naturaleza social -segregación racial, emancipación de la mujer, abuso de drogas, contaminación del medio ambiente, etc. Asimismo, dibujantes y guionistas optaron por una actitud más condescendiente hacia los superhéroes de ideología comunista, reflejando su desacuerdo con las políticas de Moscú y su afán de cooperación con sus homólogos americanos. Una orientación que volvió a modificarse durante el mandato presidencial de Ronald Reagan, cuando temas como el espionaje o la proliferación de armas nucleares adquirieron una renovada preponderancia ${ }^{86}$.

Si bien los cómics reflejaron una gran diversidad de miedos y preocupaciones que estaban presentes en la sociedad estadounidense, existieron otros productos que difundieron valores, creencias e ideologías que cuestionaban las narraciones dominantes de ese momento. En el caso de la literatura juvenil, el escaso interés que demostró la censura comunista por este tipo de libros permite comprender el auge de una producción infantil que planteaba un orden social alternativo. En otras palabras, algunos menores se convirtieron en receptores de la visión utópica defendida por la "vieja" izquierda. Una coyuntura favorecida por factores como las circunstancias personales de los propios autores -desencanto político, necesidades económicas, etc.- y la consolidación de un extenso mercado de bibliotecas escolares, que se convirtió en un instrumento eficaz para la difusión de estas obras ${ }^{87}$. A pesar de ello, estos ejemplares no fueron resultado del desarrollo de un conjunto de actividades organizadas ni pretendían inculcar una conciencia revolucionaria en los más pequeños ${ }^{88}$. Bajo la esperanza de que el sistema actual podía mejorarse a largo plazo, sus escritores eran conscientes de que las futuras generaciones necesitaban

84 Dentro de esta corriente crítica, destaca Wertham, 1954. A su vez, como consecuencia del éxito de algunos cómics estadounidenses en América Latina, a comienzos de los años setenta el crítico literario Ariel Dorfman y el sociólogo Armand Mattelart subrayaron el adoctrinamiento ideológico que sufrían los niños chilenos a través de la lectura de estas historietas infantiles. Bajo planteamientos asociados a la tesis del "imperialismo cultural", estos autores pretendían demostrar que la frontera entre la propaganda promovida desde Washington y los productos culturales de procedencia norteamericana resultaba muy difusa, pues ambos perseguían los mismos intereses de orden económico y geopolítico. Ver Dorfman - Mattelart, 1972.

85 York-York, 2012: 5.

86 Donovan, 2013: 87-88.

87 Mickenberg, 2005: 4-5 y 14-15.

88 Ibídem: 17. 
adquirir una mayor conciencia crítica para lograr suprimir las desigualdades sociales imperantes ${ }^{89}$.

\section{Conclusiones}

A lo largo de más de cuatro décadas, la historia de la infancia -y de los niños- ha ido ampliando sus líneas de investigación, poniendo en cuestión la visión utópica que había perdurado de este objeto de estudio. Mientras que han dominado aquellos trabajos centrados en una variedad de iniciativas y actividades promovidas por la esfera adulta, recientes aportaciones han demostrado cómo en ciertas ocasiones los menores de edad rebasaron el dominio dictado desde arriba y desempeñaron un importante rol como actores activos e independientes.

Dentro del ámbito académico estadounidense, uno de los campos más fructíferos de esta disciplina historiográfica ha sido el de las relaciones internacionales. Frente a la visión reduccionista que sólo valoraba la condición vulnerable e inocente de los más pequeños ante cualquier conflagración bélica, autores como Paula S. Fass, Eileen Luhr, Gabriel Rosenberg o Marcia Chatelain han planteado un escenario más rico y complejo, que permite vislumbrar la superposición de los intereses de este colectivo a cualquier objetivo político así como el establecimiento de lazos transnacionales, amparándose en un vínculo generacional.

Junto al balance sintético de este recorrido historiográfico, el artículo ha englobado algunas de las perspectivas desarrolladas en torno a esta tendencia -familia, medidas gubernamentales, iniciativas privadas, cultura de masas, etc.- bajo un eje común, el impacto de la Guerra Fría en los niños estadounidenses. A través de este caso concreto de análisis, se ha podido examinar cómo el enfrentamiento ideológico entre los Estados Unidos y la Unión Soviética logró penetrar en el marco sociocultural sobre el que se desarrollaron las experiencias de los jóvenes norteamericanos. Una aproximación a la educación, las políticas oficiales o los productos de entretenimiento de ese período, concretamente los años cincuenta y sesenta, refleja que todas estas facetas no sólo estuvieron impregnadas de las ansiedades y aspiraciones emergidas en medio de un creciente clima de tensión internacional, sino que también sirvieron como canal para la transmisión de una amplia heterogeneidad de temas y mensajes.

Frente al conocimiento que puede aportar la historia de la infancia -y de los niñossobre determinados acontecimientos y procesos históricos, muchos son los retos a superar en el futuro ante la búsqueda de unos esquemas interpretativos propios. Según Peter N. Stears, la progresiva aparición de innovadoras investigaciones no ha reducido el apego académico hacia aquellos mecanismos y representaciones concebidos por la esfera adulta, consecuencia de la escasez de fuentes primarias destinadas a otorgar voz a estos sujetos de estudio ${ }^{90}$. Análogamente, el poco énfasis en los factores de clase social, el dominio de una visión occidental que restringe el marco geográfico a examinar o la elaboración de juicios de valor desde la óptica actual son algunas de las limitaciones que todavía ensombrecen los logros conseguidos por esta disciplina. Sin duda, todos ellos representan grandes desafíos a rebasar en próximos trabajos académicos, de cuyo éxito dependerá la consolidación definitiva de este playground historiográfico.

Ibídem: 11.

90 Stearns, 2008: 35-36. 


\section{Referencias bibliográficas}

Ariès, Philippe. El niño y la vida familiar en el Antiguo Régimen. Madrid: Taurus, 1987.

Augustyn, Frederick J. Dictionary of Toys and Games in American Popular Culture. New York: Haworth Reference Press, 2004.

Barksdale, Barbara. Brainpower for the Cold War. The Sputnik Crisis and National Defense Education Act of 1958. Westport: Greenwood Press, 1981.

Casavantes, Anita. "'La Niña Adorada del Mundo Socialista': The Politics of Childhood and U.S.-Cuba-U.S.S.R. Relations, 1959-1962". Diplomatic History, vol. 42, nº 2 (2015), 1-31. DOI: 10.1093/dh/dhv001.

Chambliss, Julian C. "Upgrading the Cold War Framework: Iron Man, the Military Industrial Complex, and American Defense". En Ages of Heroes, Eras of Men. Superheroes and the American Experience, editado por Chambliss, Julian Svitavsky, William - Donaldson, Thomas. Newcastle upon Tyne: Cambridge Scholars Publishing, 2013, 163-181.

Chatelain, Marcia. "International Sisterhood: Cold War Girl Scouts Encounter the World". Diplomatic History, vol. 38, no 2 (2014), 261-270.

Chudacoff, Howard P. Children at Play. An American History. New York: New York University Press, 2007.

Cross, Gary. Kid's Stuff. Toys and the Changing World of American Childhood. Cambridge: Harvard University Press, 1997.

Delgado Gómez-Escalonilla, Lorenzo. "La diplomacia pública de Estados Unidos. Una perspectiva histórica". Revista Complutense de Historia de América, vol. 40 (2014), 277-301.

Delmont, Matthew. The Nicest Kids in Town: American Bandstand, Rock 'N' Roll, and the Struggle for Civil Rights in 1950s Philadelphia. Berkeley: University of California Press, 2012.

DeMause, Lloyd (ed.) The History of Childhood. New York: The Psychohistory Press, 1974.

Demos, John. A Little Commonwealth: Family Life in Plymouth Colony. New York: Oxford University Press, 1970.

Devlin, Rachel. Relative Intimacy: Fathers, Adolescent Daughters, and Postwar American Culture. Chapel Hill: The University of North Carolina Press, 2005.

DiPaolo, Marc. War, Politics and Superheroes. Jefferson: McFarland \& Company, 2011.

Donovan, John. 'Cold War in Comics: Clobberin' Commies and Promoting Nationalism in American Comics". En Ages of Heroes, Eras of Men. Superheroes and the American Experience, editado por Chambliss, Julian - Svitavsky, William - Donaldson, Thomas. Newcastle upon Tyne: Cambridge Scholars Publishing, 2013, 55-91.

Dorfman, Ariel - Mattelart, Armand. Para leer al Pato Donald. Comunicación de masas y colonialismo. Buenos Aires: Siglo XXI, 1972.

Dubinsky, Karen. "Children, Ideology, and Iconography: How Babies Rule the World". Journal of the History of Childhood and Youth, vol. 5, $\mathrm{n}^{\circ}$ 1(2012), 5-13.

Endy, Christopher. Cold War Holidays: American Tourism in France. Chapel Hill: The University of North Carolina Press, 2004. 
Fass, Paula S. Children of a New World. Society, Culture and Globalization. New York: New York University Press, 2007.

— "The World Is at Our Door: Why Historians of Children and Childhood Should Open Up". Journal of the History of Children and Youth, vol. 1, n ${ }^{\circ} 1$ (2008), 11-31.

- "Intersecting Agendas: Children in History and Diplomacy". Diplomatic History, vol. 38, nº 2 (2014), 294-298.

Fass, Paula S. - Grossberg, Michael (eds.). Reinventing Childhood after World War II. Philadelphia: University of Pennsylvania Press, 2011.

Field, Christopher B. "He Was a Living Breathing Human Being': Harvey Kurtzman's War Comics and the 'Yellow Peril'". En Comic Books and the Cold War, 1946-1962: Essays on Graphic Treatment of Communism, the Code and Social Concerns, editado por York, Rafiel - York, Chris. London: McFarland \& Company, 2012, 45-54.

Forman-Brunell, Miriam. "Barbie in 'LIFE': The Life of Barbie". Journal of the History of Children and Youth. Baltimore, vol. 2, n 3 (2009), 303-311.

Foster, Stuart J. Red Alert! Educators Confront the Red Scare in American Public Schools, 1947-1954. New York: Peter Lang, 2000.

García-Hoz Rosales, María Concepción. “¿A qué jugábamos? El juguete industrial en las colecciones del Museo Nacional de Antropología”. Anales del Museo Nacional de Antropología. Nos-Otros, no 3 (1996), 89-108.

Gienow-Hecht, Jessica C. E. (ed.). Music and International History in the Twentieth Century. New York - Oxford: Berghahn Books, 2015.

Grew, Raymond. "Childhood". En The Palgrave Dictionary of Transnational History. From the Mid-19th Century to the Present Day, editado por Iriye, Akira - Saunier, Pierre-Yves. Basingstoke: Palgrave Macmillan, 2009, 122-125.

Hartman, Andrew. Education and the Cold War. The Battle for the American School. New York: Palgrave Macmillan, 2008.

Hawes, Joseph M. Children in Urban Society: Juvenile Delinquency in NineteenthCentury America. New York: Oxford University Press, 1971.

Hawes, Joseph M. - Hiner, N. Ray. "Hidden in Plain View. The History of Children (and Childhood) in the Twenty-First-Century". Journal of the History of Children and Youth, vol. 1, n' 1 (2008), 43-49.

Holt, Marilyn Irvin. The Orphan Trains: Placing Out in America. Lincoln: University of Nebraska Press, 1992.

- Cold War Kids. Politics and Childhood in Postwar America, 1945-1960. Lawrence: University Press of Kansas, 2014.

Honeck, Mischa - Rosenberg, Gabriel. "Transnational Generations: Organizing Youth in the Cold War". Diplomatic History, vol. 38, n' 2 (2014), 233-239.

Iriye, Akira. Global Community: The Role of. International Organizations in the Making of the Contemporary World. Berkeley: University of California Press, 2002.

Jacobs, Margaret. "Playing with Dolls". Journal of the History of Children and Youth, vol. 1, no 3 (2008), 321-328.

Javeau, Claude "Enfant, enfance(s), enfants: quel objet pour une science sociale du jeune âge?". En Sociétés et cultures enfantines, dirigido por Djamila SaadiMokrane. Lille: Université Lille III, 2000, 25-29.

Kett, Joseph F. Rites of Passage: Adolescence in America 1790 to the Present. New York: Basic Books, 1977. 
Kordas, Ann Marie. The Politics of Childhood in Cold War America. London: Pickering \& Chatto, 2013.

Kotek, Joël. "Youth Organizations as a Battlefield in the Cold War". En The Cultural Cold War in Western Europe, 1945-1960, editado por Scott-Smith, Giles Krabbendam, Hans. London: Frank Cass Publishers, 2003, 168-191.

Lassonde, Stephen. "Ten Is the New Fourteen: Age Compression and 'Real' Childhood". En Reinventing Childhood after World War II, editado por Fass, Paula S. - Grossberg, Michael. Philadelphia: University of Pennsylvania Press, 2011, 51-67.

Lindenmeyer, Kriste. The Greatest Generation Grows Up. American Childhood in the 1930s. Chicago: Ivan R. Dee Publisher, 2005.

Lindsay, Lisa. Captives as Commodities: The Transatlantic Slave Trade. New Jersey: Pearson, 2008.

Luhr, Eileen. "Cold War Teenitiative: American Evangelical Youth and the Developing World in the Early Cold War". Journal of the History of Childhood and Youth, vol. 8, $\mathrm{n}^{\mathrm{o}} 2$ (2015) 295-317.

Magnussen, Anne - Christiansen, Hans-Christian (eds.). Comics \& Culture. Analytical and Theoretical Approaches to Comics. Copenhagen: University of Copenhagen, 2009.

Mickenberg, Julia L. Learning from the Left. Children's Literature, the Cold War, and Radical Politics in the United States. Oxford: Oxford University Press, 2005.

Mintz, Steven "The Changing Face of Children's Culture". En Reinventing Childhood after World War II, editado por Fass, Paula S. - Grossberg, Michael. Philadelphia: University of Pennsylvania Press, 2011, 38-50.

Nasaw, David. Children of the City: At Work and at Play. New York: Oxford University Press, 1985.

Ngai, Mae. Impossible Subjects: Illegal Aliens and the Making of Modern America. Princeton: Princeton University Press, 2003.

Ossian, Lisa L. The Forgotten Generation: American Children and World War II. Columbia: University of Missouri, 2011.

Oyen, Meredith. The Diplomacy of Migration: Transnational Lives and the Making of U.S.-Chinese Relations in the Cold War. Ithaca: Cornell University Press, 2015.

Peacock, Margaret. Innocent Weapons. The Soviet and American Politics of Childhood in the Cold War. Chapel Hill: The University of North Carolina Press, 2014.

Reid, Jason. “'My Room! Private! Keep Out! This Means You!': A Brief Overview of the Emergence of the Autonomous Teen Bedroom in Post-World War II America”. Journal of the History of Children and Youth, vol. 5, n 3 (2012), 419443.

Rosenberg, Gabriel. Breeding the Future: The American 4-H Movement and the Roots of the Modern Rural World, 1914-1948. Providence: Brown University, 2011.

Schweinitz, Rebecca de. If We Could Change the World: Young People and America's Long Struggle for Racial Inequality. Chapel Hill: The University of North Carolina Press, 2009.

Scott, David C. - Murphy, Brendan. The Scouting Party. Pioneering and Preservation Progressivism and Preparedness in the Making of the Boy Scouts of America. Dallas: Red Honor Press, 2010. 
Sharp, Charles Dee. The Wonder of American Toys. North Haven: Linnet Books, 2002.

Shorter, Edward. The Making of the Modern Family. New York: Basic Books, 1975.

Stearns, Peter N. "Challenges in the History of Childhood". Journal of the History of Children and Youth, vol. 1, no 1 (2008), 35-42.

Stone, Lawrence. The Family, Sex and Marriage in England, 1500-1800. New York: Harper \& Row, 1977.

Suárez-Orozco, Carola - Suárez-Orozco, Marcelo M. Children of Immigration. Cambridge: Harvard University Press, 2001.

Thompson, Antonio S. "Nationalism and Power: Captain America, Governmental Policy, and the Problem of American Nationalism". En Ages of Heroes, Eras of Men. Superheroes and the American Experience, editado por Chambliss, Julian - Svitavsky, William - Donaldson, Thomas. Newcastle upon Tyne: Cambridge Scholars Publishing, 2013, 106-120.

Wagg, Stephen - Andrews, David L. (eds.). East Plays West: Sport and the Cold War. London: Routledge, 2006.

Wertham, Frederic. Seduction of the Innocent. New York: Rinehart \& Company, 1954.

Wills, Chuck. Boy Scouts of America. A Centennial History. New York: DK Publishing, 2009.

Yans-McLaughlin, Virginia. Family and Community. Italian Immigrants in Buffalo, 1880-1930. Ithaca: Cornell University Press, 1977.

York, Rafiel. "The Fantastic Four: A Mirror of Cold War America". En Comic Books and the Cold War, 1946-1962: Essays on Graphic Treatment of Communism, the Code and Social Concerns, editado por York, Rafiel - York, Chris. London: McFarland \& Company, 2012, 204-215.

York, Rafiel - York, Chris. "Introduction: Frederic Wertham, Containment, and Comic Books". En Comic Books and the Cold War, 1946-1962: Essays on Graphic Treatment of Communism, the Code and Social Concerns, editado por York, Rafiel - York, Chris. London: McFarland \& Company, 2012, 5-15. 